\title{
PHOTOELECTRIC OCCULTATION OBSERVATIONS OF \\ REGULUS AND THE PLEIADES
}

\author{
RICHARD A. BERG \\ Department of Physics, University of Delaware, Newark, Delaware, U.S.A.
}

The dual channel photometer is mounted on the tailpiece of the 32-in. $f / 16$ reflector at McCormick Observatory's Fan Mountain Station. A 50-50 beam splitting mirror distributes the light to two EMI 6256 S/A photomultipliers. A focal plane diaphragm admits an area of sky of $100 \mathrm{sec}^{2}$ of arc, and UBV or narrow-band filters are used to isolate spectral regions. The photoelectrons are fed through preamplifiers and amplifiers to a specially designed integrated-circuit photon counting instrument. The preamplifiers are attached as closely as possible to the photomultiplier anodes, while the remaining circuits are housed separately. A Sony TC- 200 stereo tape recorder is used to record the output signal from the counter, and an oscilloscope is used for real-time monitoring of the counter operation during occultations.

In Figure 1 the interconnection of logical units of the pulse counter is illustrated. This instrument consists of a 12-bit binary counter, a 12-bit storage register, a crystal

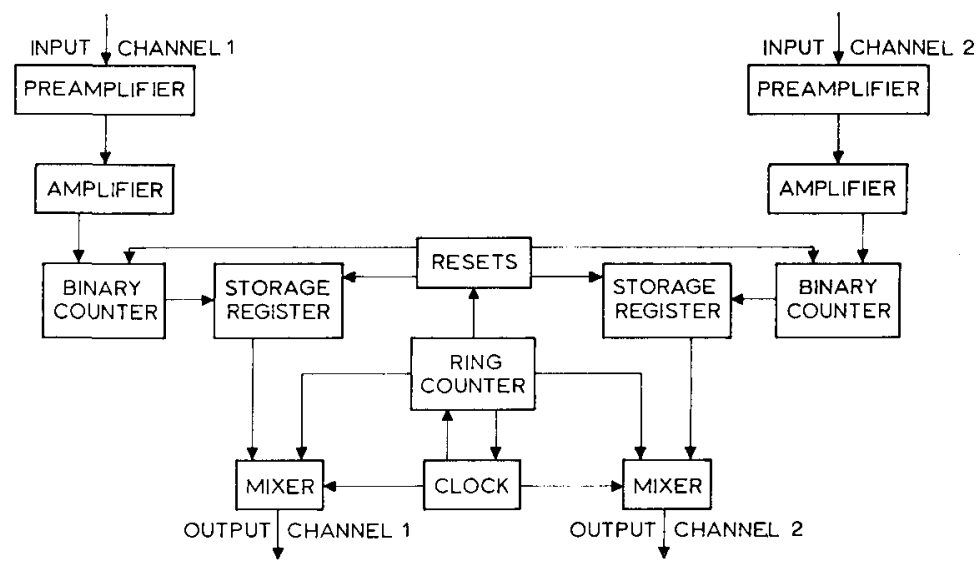

Fig. 1. Interconnection of logical units of the photon counter.

oscillator clock, and other logic circuits necessary for proper operation. The normal integration time is $1.04 \mathrm{msec}$, but this can be increased by factors of two up to 1.06 sec. A clock signal, at $1.04 \mathrm{msec}$ intervals, causes three events to occur in turn. First the storage register is cleared; then the counter bit configuration is shifted to storage; and finally the counter is cleared and continues counting from zero. While the counter is accumulating for the next cycle, the storage register bits are read out sequentially as an amplitude modulated $12 \frac{1}{2} \mathrm{kHz}$ signal and are recorded on the tape. 
The counter itself operates linearly up to rates exceeding $12 \mathrm{MHz}$. when fed with equally spaced narrow pulses; but, of course, the pulses from a photomultiplier are neither uniform in height or width nor equally spaced. This system combines the photon counting capability of a multichannel analyzer, with the data storage capacity of a large computer. In addition, the cost of all hardware for the photon counter is less than $\$ 400$. For this modest price, however, one must expend a large amount of personal labor to convert the data to machine readable form.

Photon counts vs. time are recovered from the tape and represent the basic data. These occultation curves are restored to strip brightness distribution curves, or intensity profiles, by a deconvolution technique (Scheuer, P. A. G.: 1962, Australian J. Phys. 15, 333; Von Hoerner, S.: 1964, Astrophys. J. 140, 65). To use this technique one convolves the observed occultation curve with functions of increasingly smaller widths until a resolution limit is reached. Produced at each step is an intensity profile of the source as if it had been scanned by a fan beam of increasingly smaller angular beamwidth.

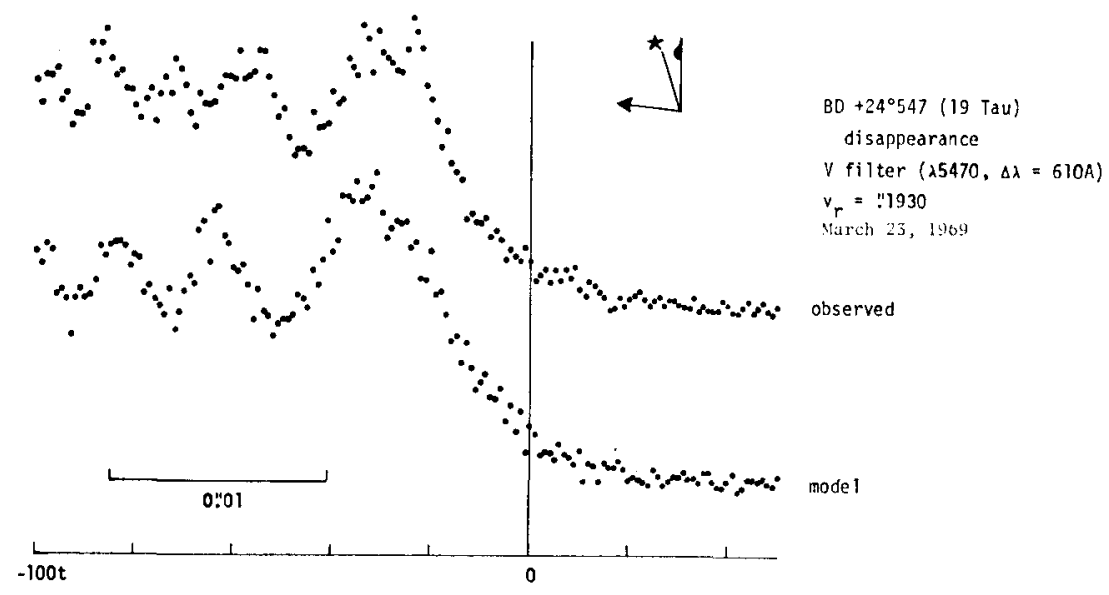

Fig. 2. The occultation and model curve of $\mathrm{BD}+24^{\circ} 547$, V-color

Figure 2 shows an observed occultation curve of 19 Tauri and a model curve for the same occultation generated from the prediction parameters. The abscissa indicates channel number, or relative elapsed time proceeding left to right. Each unit of ' $t$ ' is $1.04 \mathrm{msec}$. The figure in the upper corner indicates celestial north by a flag, the direction of the velocity of the Moon by an arrow, and the position angle of the star on the limb by a star. The velocity component of the lunar limb at the position angle of the star is given in arcseconds per second as $v_{r}$.

19 Tauri was observed through a $610 \mathrm{~A}$ wide Johnson V filter; the model curve assumes a monochromatic pointsource. The difference in the observed and model fringe spacings is attributed to a lunar slope, to which near-grazing occultations such as this are particularly sensitive. Figure 3 shows the Johnson B-color observation and 


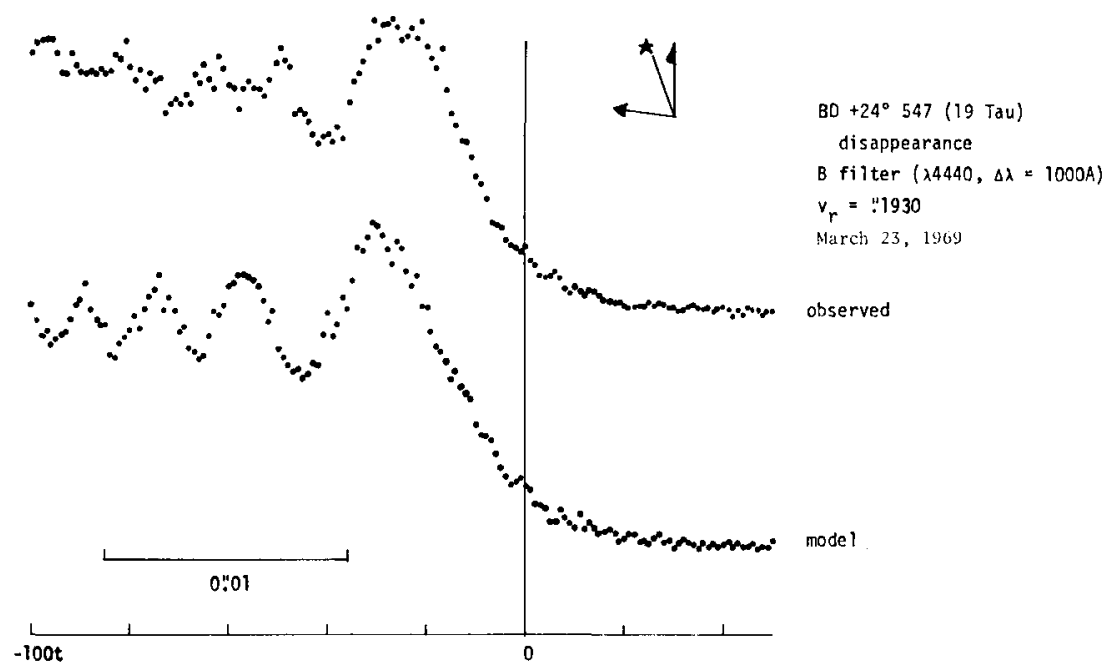

Fig. 3. The occultation and model curve of $\mathrm{BD}+24^{\circ} 547$, B-color.

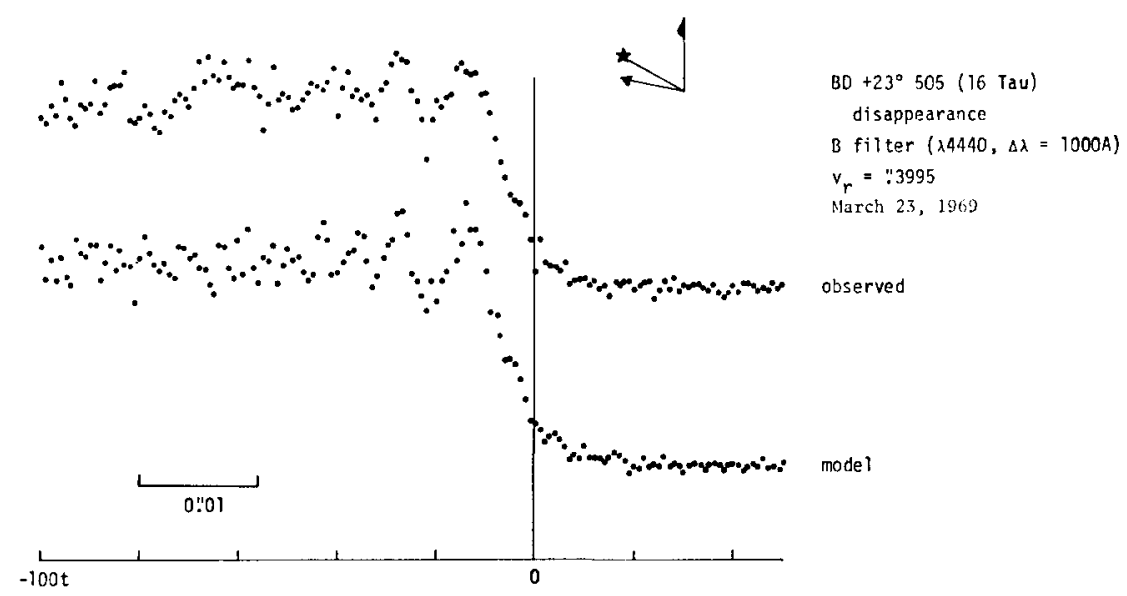

Fig. 4. The occultation and model curve of $\mathrm{BD}+23^{\circ} 505$.

model of the same occultation. From these two observations, the lunar slope at the point of disappearance is computed to be $4.1 \pm 0^{\circ} .1$.

The observation and model occultation of 16 Tauri is shown in Figure 4. The model is constructed assuming two monochromatic point-sources separated by 6 milliarcseconds (masec) having a $\Delta B$ of 2 . These values are determined from the restoration curves, but it is most instructive to show the 'recomputed' observation against the observed curve. The fringe spacings agree very well. The only unusual feature seen is a spurious dip in the observed curve at $-80 t$. In Figure 5 the restored intensity profiles for a number of restoring beamwidths are seen. The abscissa in this figure is arcseconds, and the bars on the right indicate the restoration beamwidth. The resto- 
rations quickly reveal the secondary component at separation $6.2 \pm 0.2$ masec, in scan position angle $247^{\circ}$. A comparison of the areas under the restored profiles of each component gives a delta blue magnitude of $2.0 \pm 0.2$. The large deviations at the left of the restored profiles can be attributed to the dip occurring at a similar position on the observed occultation curve.

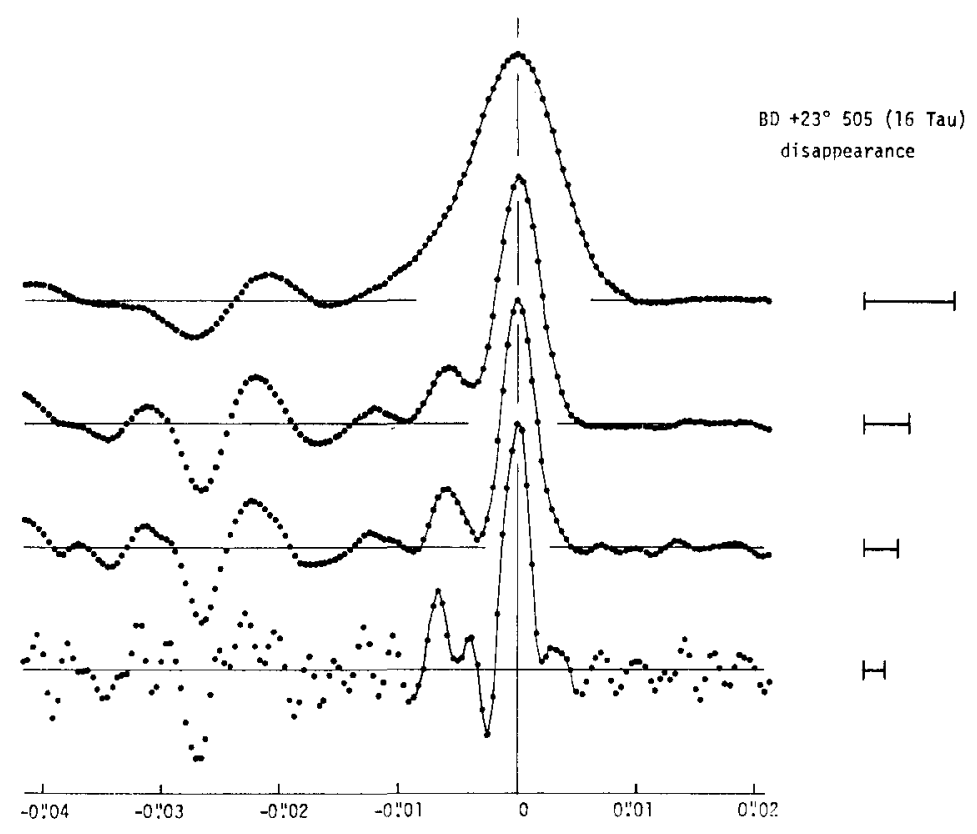

Fig. 5. Restoration intensity profiles of $\mathrm{BD}+23^{\circ} 505$.

Figure 6 shows the occultation and model of 24 Tauri, a $6^{\mathrm{m}} .2$ A0V star. Of particular interest is the absence of fringes, a characteristic of occultations of stars of larger angular diameters. Measurements of the HPFW of the restored profiles indicate an angular diameter of $10.4 \pm 1.7$ masec, over fifty times the computed blackbody diameter. The maximum of the observed curve is $30 \%$ above the preoccultation intensity, as compared with only about $10 \%$ for the model occultation of diameter 10 masec shown in the lower curve. The lunar limb velocity computed from the position of the fringe maximum is about one-half the observed velocity. A lunar slope of $22^{\circ}$ will account for this change. One Lunar Orbiter IV photograph, covering the surface which was the limb region for this occultation, shows an obviously disturbed terrain. But the $80 \mathrm{~m}$ resolution of the photograph is not adequate to see rocks or undulations on the scale of a few meters over which the occultation occurred.

In Figure 7 the unusual restored intensity profiles are seen. The restoration assumes that the predicted lunar limb rate is correct. The triplicity suggested by the profiles is probably not real in view of the similar deviations at the zero-level ends of the 
profiles. It is interesting to note that the observed occultation curve (Figure 6) can be modelled fairly well by assuming a triple source structure with delta magnitudes and separations indicated by the restored profiles.

The disappearance of Regulus at the dark limb and the model are shown in Figure 8. The observation was made through a $130 \mathrm{~A}$ interference filter centered on the

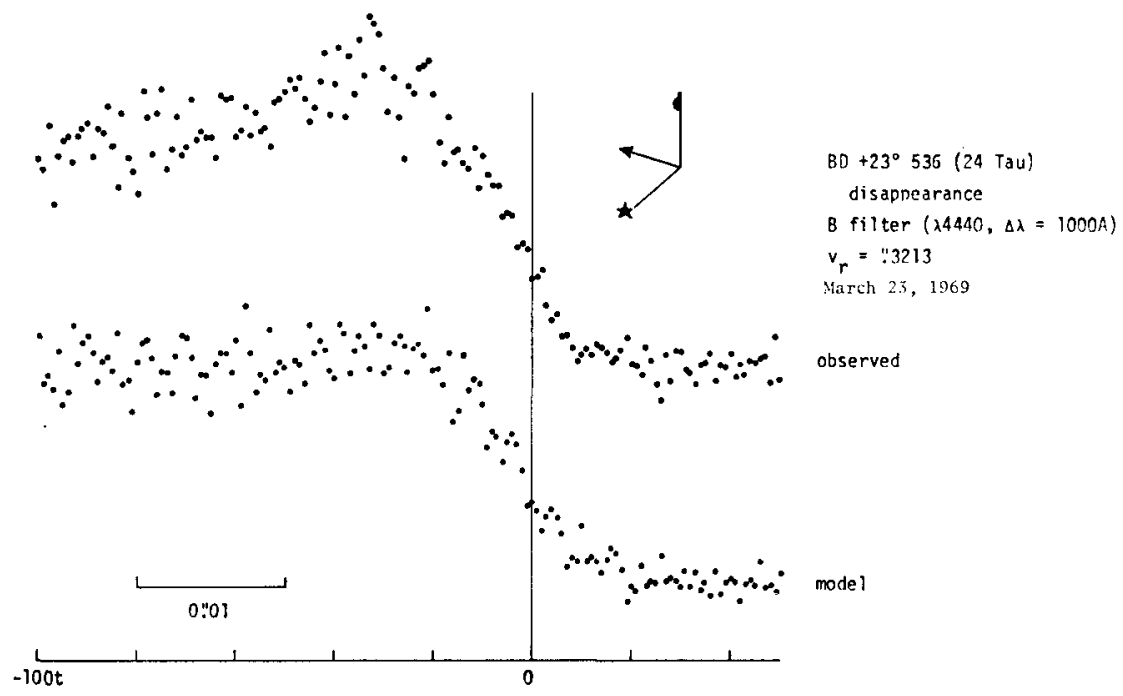

Fig. 6. The occultation and model curve of $\mathrm{BD}+23^{\circ} 536$.

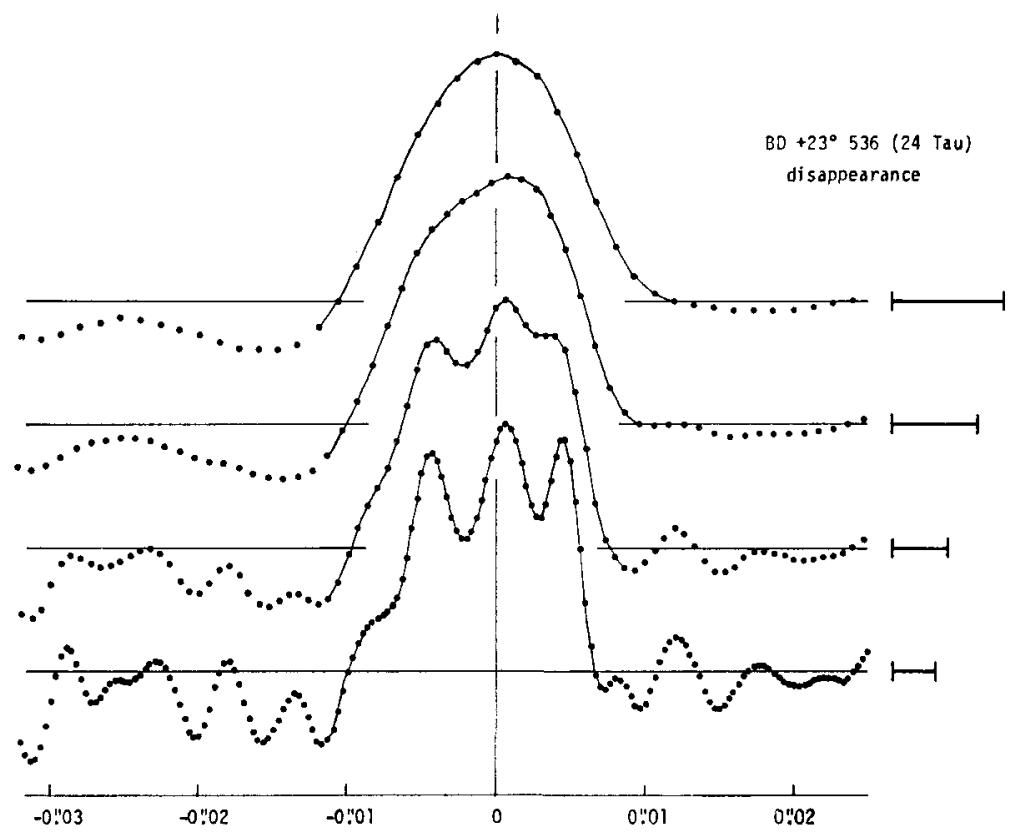

Fig. 7. Restoration intensity profiles of $\mathrm{BD}+23^{\circ} 536$. 


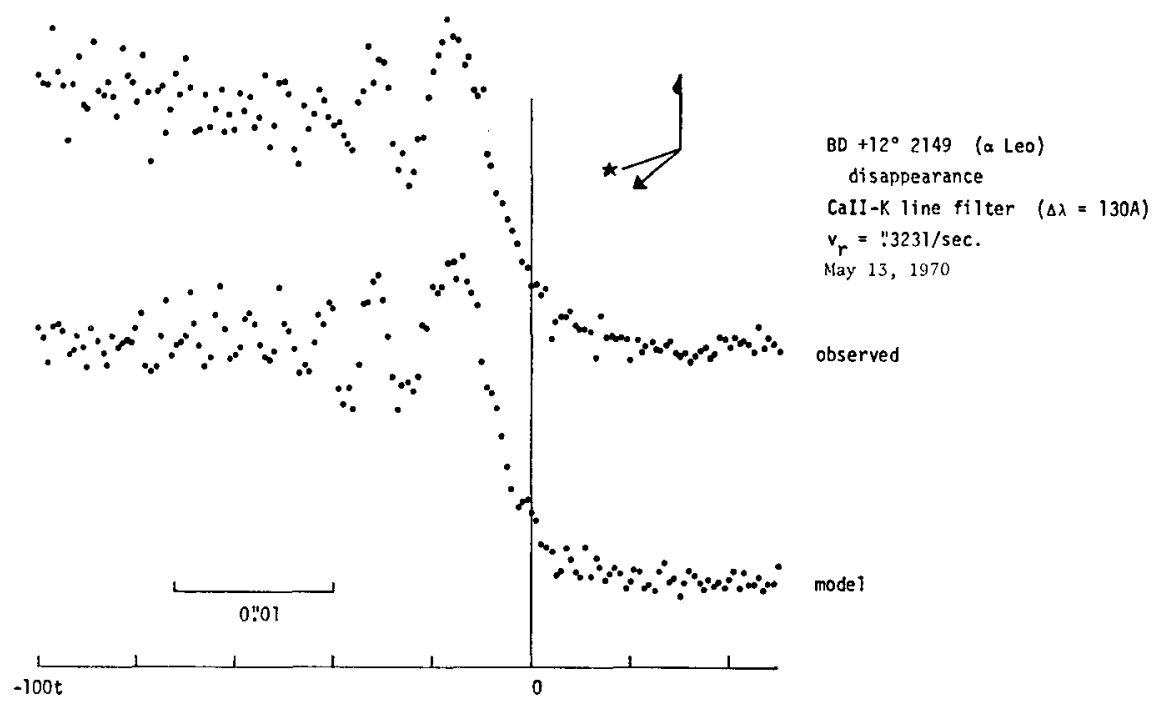

Fig. 8. The occultation and model curve of the disappearance of Regulus.

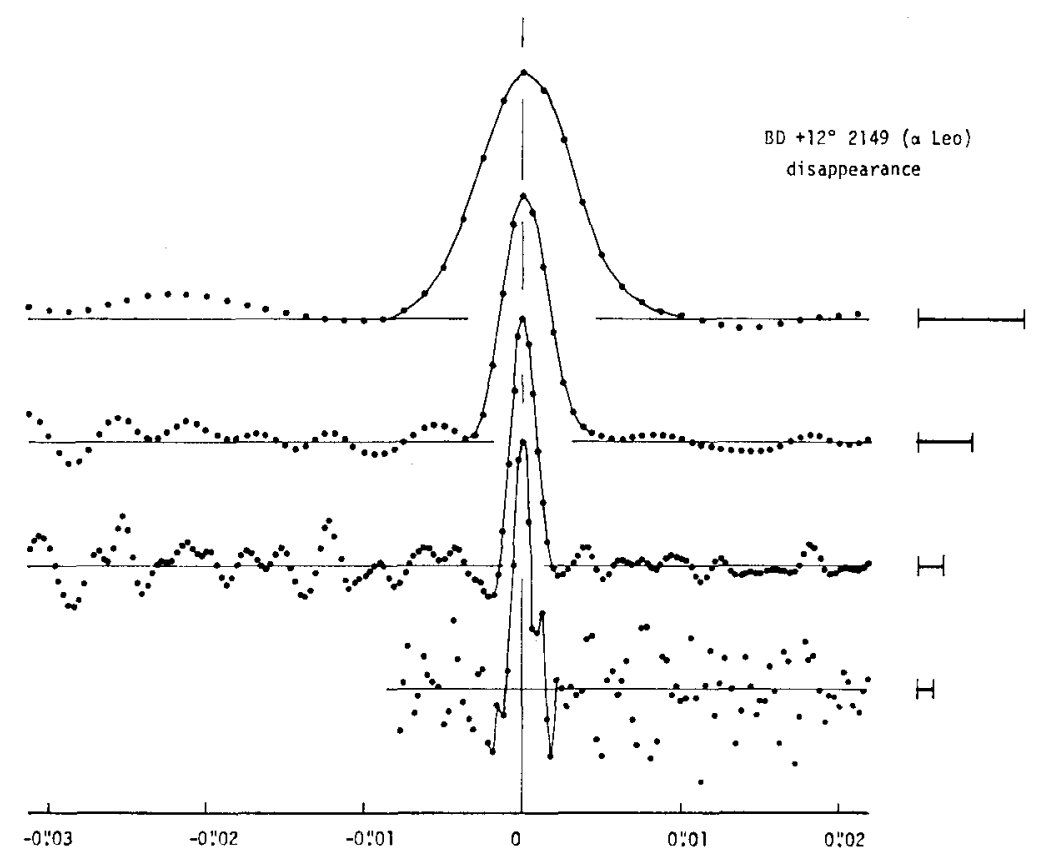

Fig. 9. Restoration intensity profiles of the disappearance of Regulus. 
$K$-line of ionized calcium. The occultation curve exhibits no unusual features and the predicted fringe spacing agrees with the observed fringe spacing. In the restored intensity profiles, which are seen in Figure 9 , there is no evidence for duplicity at scan position angle $110^{\circ}$. Measurements of the HPFW of the restored profiles indicate an upper limit to the angular diameter of Regulus of 1.1 masec.

The bright limb reappearance of Regulus is shown in Figure 10. Offset guiding was

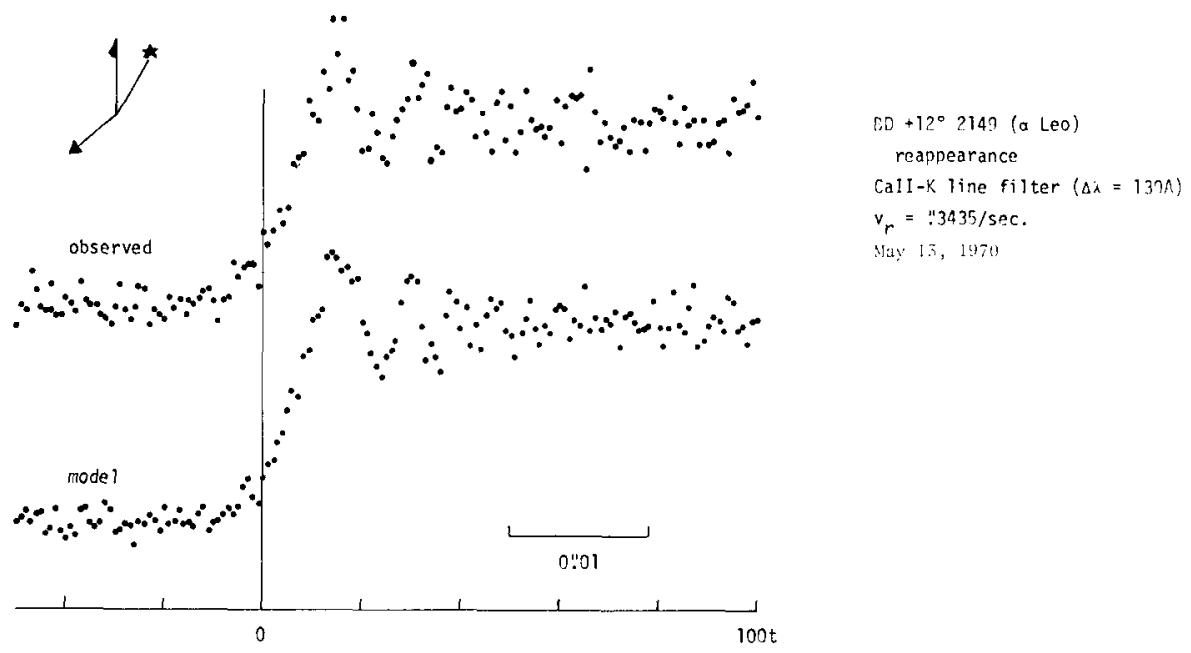

Fig. 10. The occultation and model curve of the reappearance of Regulus.

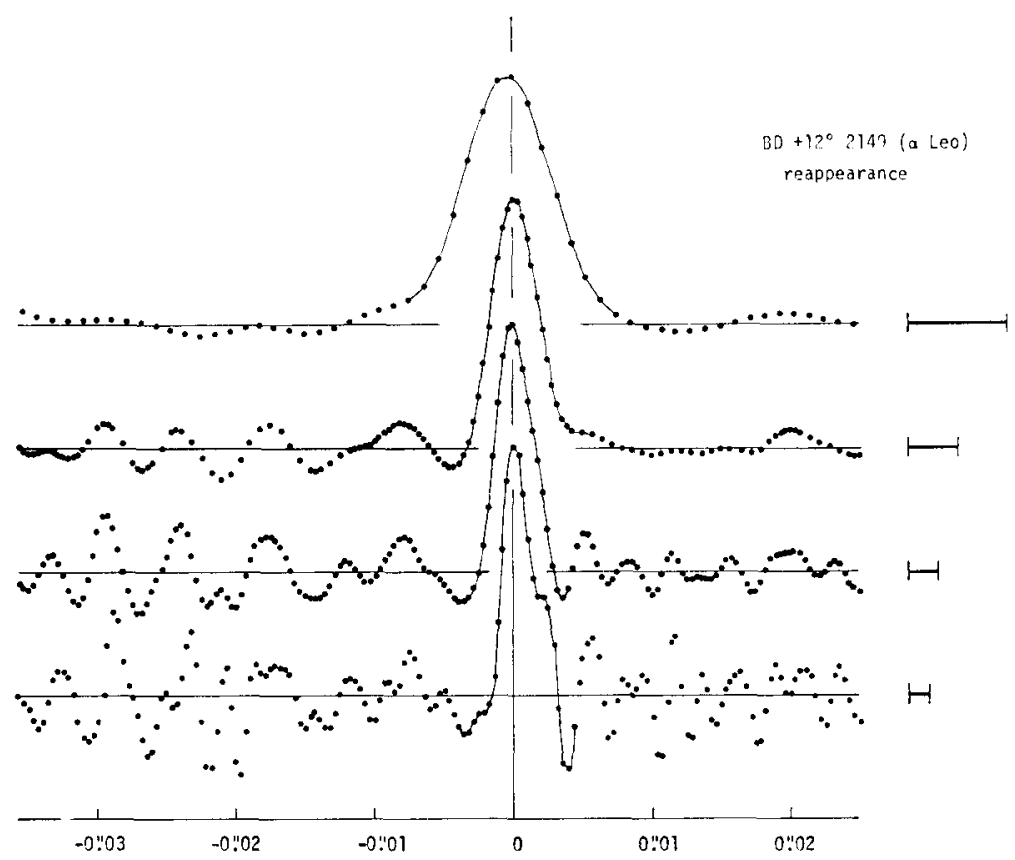

Fig. 11. Restoration intensity profiles of the reappearance of Regulus. 
used to center Regulus in the photometer diaphragm. In addition to being necessary for reappearances, offset guiding is clearly practical. The observed curve is somewhat noisier because of the contribution from the illuminated lunar limb, but again the occultation curve appears normal. In the last figure, Figure 11, the restored intensity profiles are seen. There is no evidence for duplicity at scan position angle $330^{\circ}$. The measured angular diameter of Regulus deduced from the reappearance is $1.7 \pm 0.5$ masec.

The angular diameters derived from the deconvolution of occultation curves are in no way certain. For one thing, the measurement of HPFW assumes a gaussian stellar intensity profile. Depending on the restoration beamwidth, the HPFW can be either greater than or less than the actual angular diameter. Additionally, pulse counting coincidence corrections have not been applied to these observations. These corrections will not affect the observed separation of double sources, but they will tend to reduce the observed angular diameters - perhaps by $10 \%$. Finally, limb irregularities appear to play a more significant role in determining the shape of the occultation curve than has been previously believed. The importance of obtaining multiple-color or multiple-site observations of the same occultation event, with precise timings, cannot be overstated since at the present time it is only through duplicate observations that stellar and lunar anomalies can be separated.

\section{Acknowledgement}

The author acknowledges with pleasure the support of the Leander McCormick Observatory and the University of Virginia Department of Astronomy. 